Roberto Farina

Eriberto Bressan

Andrei Taut

Alessandro Cucchi

Leonardo Trombelli

Short Communication

\title{
Plasma rich in growth factors had limited effect on early bone formation in extraction sockets
}

\author{
Response to "Anitua, E., Alkhraisat, M.H. \\ \& Orive, G. (2013) Letter to the Editor: \\ Rigorous methodology is the school of \\ coherent conclusions in science. European \\ Journal of Oral Implantology 6: 9-11."
}

\begin{abstract}
Authors' affiliations:
Roberto Farina, Leonardo Trombelli, Research Centre for the Study of Periodontal and PeriImplant Diseases, University of Ferrara, Ferrara, Italy

Eriberto Bressan, Department of Medical and Surgical Specialties, University of Padova, Padova, Italy

Andrei Taut, Department of Periodontics and Oral Medicine, School of Dentistry, University of Michigan, Ann Arbor, MI, USA

Alessandro Cucchi, Department of Clinical and Experimental Medicine, University of Ferrara, Ferrara, Italy
\end{abstract}

Corresponding author:

Leonardo Trombelli, DDS, PhD

Research Centre for the Study of Periodontal and

Peri-Implant Diseases, University of Ferrara

C.so Giovecca 203 Ferrara, 44100, Italy

Tel.: +390532 205277

Fax: +390532 202329

e-mail: leonardo.trombelli@unife.it

Date:

Accepted 3 June 2013

\section{To cite this article:}

Farina R, Bressan E, Taut A, Cucchi A, Trombelli L. Plasma rich in growth factors had limited effect on early bone formation in extraction sockets.

Clin. Oral Impl. Res. 25, 2014, 1189-1191

doi: $10.1111 /$ clr. 12230

Key words: bone regeneration, clinical research, clinical trials, growth factors, guided tissue regeneration, morphometric analysis, wound healing

\begin{abstract}
Aim: To address the criticisms raised by Anitua et al. (European Journal of Oral Implantology, 6, 2013, 9-11) to the article "Plasma Rich in Growth Factors (PRGF) in Human Post-Extraction Sockets: an Histological and Histomorphometric Study.", recently published by Farina and colleagues (Clinical Oral Implants Research 2012; doi: 10.1111/clr.12033).

Methods: All the methodological aspects criticized in the letter by Anitua et al. were thoroughly reconsidered and discussed in a structured short communication. When indicated, pertinent, additional material was included to reinforce our considerations.

Results: As most clinical studies in implant dentistry, including previous studies evaluating the efficacy/effectiveness of PRGF, the study by Farina et al. has some limitations. However, it is currently the only published controlled trial using quantitative parameters related to PRGF-induced early bone formation.

Conclusions: Despite all limitations, the results of the study by Farina et al., which were based on different quantitative parameters (micro-CT scan, immunohistochemical markers of wound healing and bone deposition), indicated a limited effect of PRGF on early bone formation in extraction sockets.
\end{abstract}

Preliminary observational studies indicated that Plasma Rich in Growth Factors (PRGF) may exert beneficial effects when applied to healing sockets (Anitua 1999; Nazaroglou et al. 2009; Mozzati et al. 2010). PRGF has been claimed to induce a faster re-epithelialization and a consistently higher content of mineralized bony trabeculae at 10-16 weeks after tooth extraction in humans (Anitua 1999). Unfortunately, the use of PRGF in extraction sockets, which has been promoted and encouraged among dental professionals over the last decade with the aim to enhance bone formation, was mainly based on qualitative assessment on histologic samples (Anitua 1999), radiographic evaluation of anecdotal cases (Nazaroglou et al. 2009) and short-term
(7 days) cytokine and protein expression (Mozzati et al. 2010).

We are perfectly aware that our study (Farina et al. 2012) has clear limitations as most clinical studies in implant dentistry /Cairo et al. 2012; Meijer \& Raghoebar 2012; Rocchietta \& Nisand 2012; Schwarz et al. 2012; Vignoletti \& Abrahamsson 2012), including studies evaluating the efficacy/effectiveness of PRGF. According to the regulatory authorities, there are standard operating procedures on patient recruitment, randomization, sample size calculation, selection of primary and secondary endpoints, examiner calibration, etc. We have to accept that in research reality, it is often hard to consider all these and even more aspects of study design. Despite 
all the limitations, our study is currently the only controlled trial using quantitative parameters related to PRGF-induced early bone formation. The results, based on different quantitative parameters (micro-CT scan, immunohistochemical markers of wound healing and bone deposition), indicated a limited effect of PRGF on early bone formation in extraction sockets.

With respect to the issues raised by Anitua et al. (2013):

1). As clearly stated in the Materials and Methods, our study was designed as a parallel-arm, open-label expertise-based controlled clinical trial. A clinical operator with specific training and clinical skill as to the treatment with PRGF selected all PRGF-treated sites, whereas two trained clinical operators selected all control sites (i.e., extraction sites left to spontaneous healing). The choice of this experimental (non-randomized) design has been made to optimize the outcomes of PRGF treatment because PRGF is an operator-sensitive technique with a specific learning curve.

2). Surgical treatment in PRGF sockets was undertaken by an operator with extensive experience with this technology. Sutures were placed according to the clinician's judgment. To the best of our knowledge, there is no evidence supporting the need for additional PRGF-Endoret membrane to allow for PRGF to exert its effects on bone formation. Thus, the relevance of a primary wound closure and the additional effect of a PRGF-Endoret membrane still need to be demonstrated.

3). The final statement of the abstract has been written according to the study results. We are aware that a subanalysis to explore the role of smoking on PRGFinduced healing dynamics would have added novel information /data on the potential detrimental effect of smoking on the quality, as assessed histomorphometrically, of extraction wound healing during the early phases are still lacking) as well as allowed for a better interpretation of our findings. Unfortunately, this was not feasible due to the limited sample size of subgroups (with respect to smoking status, treatment and observation interval). The current level of evidence (including the references quoted by Anitua in its letter) only suggests a potential detrimental effect of smoking. In a recent study, no statistically significant differences were found in the tissue composition between healed extraction sites in smoker and non-smoker patients (Lindhe et al. 2012).

4). In our study, the cause for tooth extraction was recorded but not taken into account in the analysis. Recently, Lindhe et al. reported no differences in the tissue composition of post-extraction sites between advanced periodontitis and nonperiodontitis groups (Lindhe et al. 2012). The evidence brought by Ahn \& Shin (2008) of a delayed bone healing phase of extraction sockets in advanced periodontitis patients seems to point out the relevance of other factors (e.g., incidence of loss of the socket bone walls and location of the extraction sockets with respect to tooth types) rather than the periodontal infection per se in affecting the rate of bone formation between diseased and healthy sockets.

5). In our study, periodontal disease was not considered as an inclusion/exclusion criteria and patients were enrolled irrespective of their periodontal status. In line with our protocol, previous studies evaluat- ing PRGF treatment in extraction sockets included patients with teeth extracted for "severe periodontal disease" (Anitua 1999). To the best of our knowledge, there is no evidence supporting the hypothesis that the individual susceptibility to periodontitis may exert an effect on the healing of extraction sockets (Farina \& Trombelli 2012). Consistently, a recent study failed to find significant differences in the tissue composition of fully healed extraction sites of periodontitis and non-periodontitis subjects (Lindhe et al. 2012).

6). Recent evidence showed a similar healing pattern of extraction sites among different locations within the dentition (Lindhe et al. 2012). In our study, the effect of PRGF was merely investigated in post-extraction sockets from singlerooted teeth or single roots of mandibular molars. Although such selection may have reduced the inter-site differences in healing dynamics due to the variability in socket dimension, treatment of defects of limited size may have masked and

(a)

Micro CT - T1 -
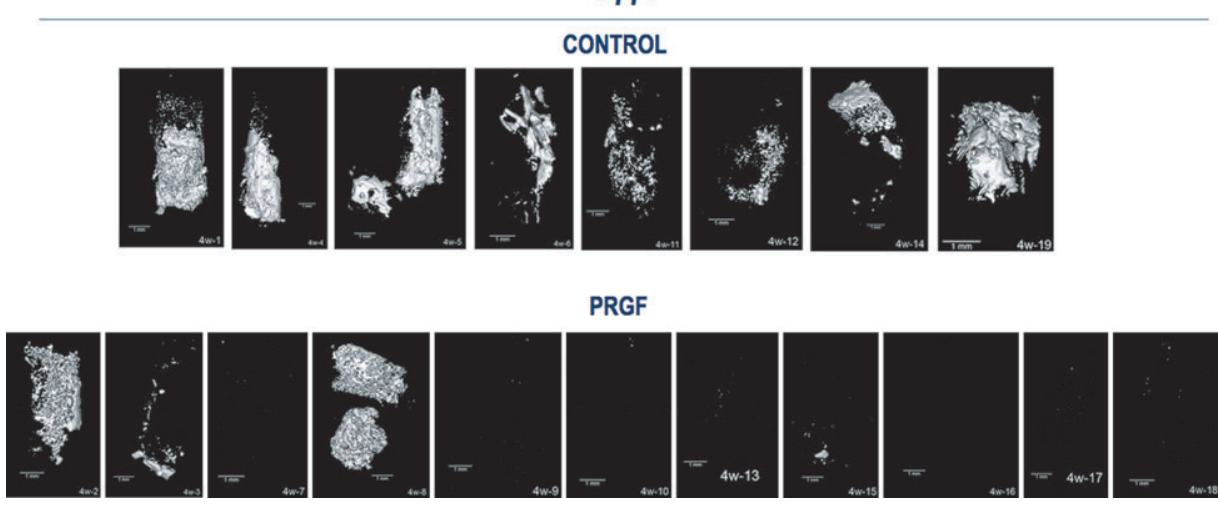

(b)

Micro CT

- T2 -
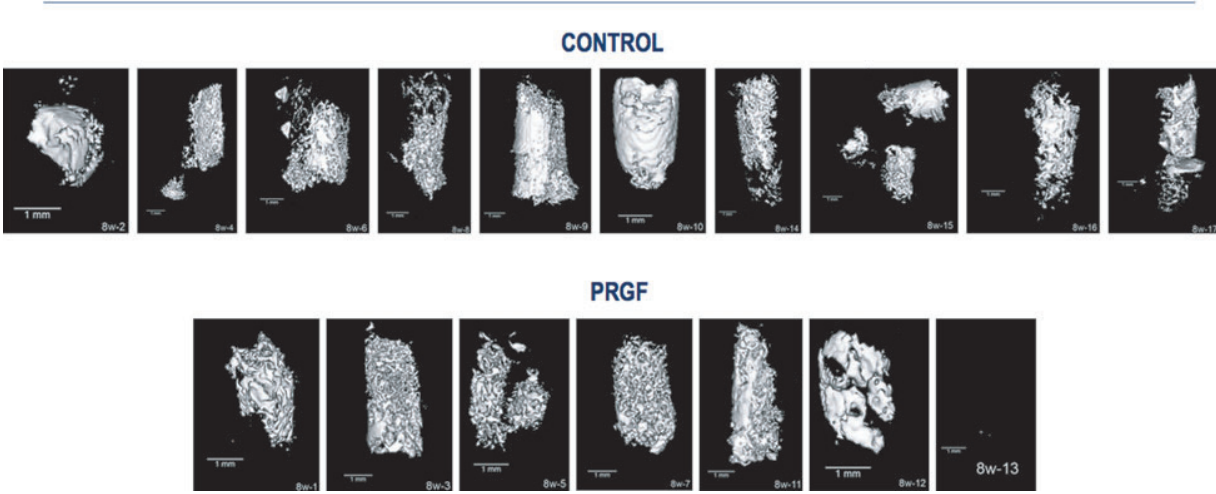

PRGF

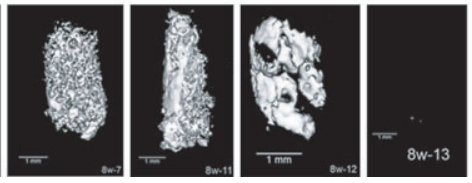

Fig. 1. Micro-CT scans of all study specimens at T1 (4-6 weeks) (a) and T2 (7-10 weeks) (b) for PRGF and control groups. 
underestimated the true biologic potential of PRGF on early bone formation. Further controlled studies are needed to assess which type of post-extraction conditions, including patient characteristics as well as dimension and morphology of the socket, may represent an indication for the additional use of PRGF.

7). While the study by Trombelli et al. (2008) reported an increase in osteocalcin (OCN) staining from 2-4 weeks to 6-8 weeks, Farina et al. (2012) indicated a statistically significant decrease in OCN staining from T1 (4-6 weeks) to T2 (7-10 weeks). It is important to consider that different methods of OCN quantification were used by Trombelli et al. (2008) and Farina et al. (2012). In the 2008 study, the OCN score was related to the percentage of the periphery of the woven bone projections that showed the presence of OCN-stained cells. In the 2013

\section{References}

Ahn, J.J. \& Shin, H.I. (2008) Bone tissue formation in extraction sockets from sites with advanced periodontal disease: a histomorphometric study in humans. International Journal of Oral and Maxillofacial Implants 23: 1133-1138.

Anitua, E. (1999) Plasma rich in growth factors: preliminary results of use in the preparation of future sites for implants. International Journal of Oral and Maxillofacial Implants 14: 529-535.

Anitua, E., Alkhraisat, M.H. \& Orive, G. (2013) Letter to the Editor: rigorous methodology is the school of coherent conclusions in science. European Journal of Oral Implantology 6: 9-11.

Cairo, F., Sanz, I., Matesanz, P., Nieri, M. \& Pagliaro, U. (2012) Quality of reporting of randomized clinical trials in implant dentistry. A systematic review on critical aspects in design, outcome assessment and clinical relevance. Journal of Clinical Periodontology 39(Suppl 12): 81-107.

Chang, P.C., Seol, Y.J., Kikuchi, N., Goldstein, S.A. \& Giannobile, W.V. (2010) Functional apparent moduli as predictors of oral implant osseointegration dynamics. Journal of Biomedical Materials Research. Part B. Applied Biomaterials 94: 118126. Erratum in: Journal of Biomedical Materials Research. Part B. Applied Biomaterials 2010;95: 481.

Dunn, M.D., Park, C.H., Kostenuik, P.J., Kapila, S. \& Giannobile, W.V. (2007) Local delivery of osteoprotegerin inhibits mechanically mediated bone modeling in orthodontic tooth movement. Bone 41: 446-455.

Farina, R., Bressan, E., Taut, A., Cucchi, A. \& Trombelli, L. (2012) Plasma rich in growth factors in human extraction sockets: a radiographic and histomorphometric study on early bone deposition. Clinical Oral Implants Research doi: 10. 1111/clr.12033. study, average pixel intensity was used to quantify the relative expression of OCN in standardized regions of interest in the coronal, middle and apical portions of each sample, and the OCN score was inversely correlated to the average pixel intensity.

8). Due to the nature of the study purpose (to determine whether and to what extent the additional application of PRGF to an extraction socket may influence the early bone deposition), bone volume was regarded as the primary outcome variable. As data on bone volume to evaluate the healing process of human extraction sockets (either treated with PRGF or not) could not be retrieved, the post hoc calculation was the only option to obtain information on the statistical power of the study. The issue of statistical power has been thoroughly evaluated by expert statistical referees during the revision process before publication.

9). The choice of micro-CT was based on its sensitivity for hard tissue assessment, capable to detect minimal differences between treatment groups (Dunn et al. 2007; Jin et al. 2007; Park et al. 2007, 2012; Rogers et al. 2007; Chang et al. 2010; Neiva et al. 2011). The use of this (non-invasive) technology also prevents an excessive loss of material for each sample to be processed for immunohistochemical staining. Thus, we still regard the use of micro-CT analysis as an added value in the scientific methodology adopted. To provide a broader overview of the diagnostic potential of this technology, we include Fig. 1a,b which show the micro-CT scans of all study specimens at $\mathrm{T} 1$ and $\mathrm{T} 2$, respectively, for PRGF and control groups.
Farina, R. \& Trombelli, L. (2012) Wound healing of extraction sockets. Endodontics Topics 25: 16-43.

Jin, Q., Cirelli, J.A., Park, C.H., Sugai, J.V., Taba, M. Jr, Kostenuik, P.J. \& Giannobile, W.V. (2007) RANKL inhibition through osteoprotegerin blocks bone loss in experimental periodontitis. Journal of Periodontology 78: 1300-1308.

Lindhe, J., Cecchinato, D., Bressan, E., Toia, M., Araújo, M.G. \& Liljenberg, B. (2012) The alveolar process of the edentulous maxilla in periodontitis and non-periodontitis subjects. Clinical Oral Implants Research 23: 5-11.

Meijer, H.J. \& Raghoebar, G.M. (2012) Quality of reporting of descriptive studies in implant dentistry. Critical aspects in design, outcome assessment and clinical relevance. Journal of Clinical Periodontology 39(Suppl 12): 108-113.

Mozzati, M., Martinasso, G., Pol, R., Polastri, C., Cristiano, A., Muzio, G. \& Canuto, R. (2010) The impact of plasma rich in growth factors on clinical and biological factors involved in healing processes after third molar extraction. Journal of Biomedical Materials Research. Part A. 95: 741-746.

Nazaroglou, I., Stavrianos, C., Kafas, P., Matoulas, E., Upile, T., Barlas, I. \& Jerjes, W. (2009) Radiographic evaluation of bone regeneration after the application of plasma rich in growth factors in a lower third molar socket: a case report. Cases Journal 3: 9134.

Neiva, R., Pagni, G., Duarte, F., Park, C.H., Yi, E., Holman, L.A. \& Giannobile, W.V. (2011) Analysis of tissue neogenesis in extraction sockets treated with guided bone regeneration: clinical, histologic, and micro-CT results. International Journal of Periodontics and Restorative Dentistry 31: 457-469.

Park, C.H., Abramson, Z.R., Taba, M. Jr, Jin, Q., Chang, J., Kreider, J.M., Goldstein, S.A. \& Gian- nobile, W.V. (2007) Three-dimensional microcomputed tomographic imaging of alveolar bone in experimental bone loss or repair. Journal of Periodontology 78: 273-281.

Park, C.H., Rios, H.F., Jin, Q., Sugai, J.V., PadialMolina, M., Taut, A.D., Flanagan, C.L., Hollister, S.J. \& Giannobile, W.V. (2012) Tissue engineering bone-ligament complexes using fiber-guiding scaffolds. Biomaterials 33: 137-145.

Rocchietta, I. \& Nisand, D. (2012) A review assessing the quality of reporting of risk factor research in implant dentistry using smoking, diabetes and periodontitis and implant loss as an outcome: critical aspects in design and outcome assessment. Journal of Clinical Periodontology 39/Suppl 12): 114-121.

Rogers, J.E., Li, F., Coatney, D.D., Rossa, C., Bronson, P., Krieder, J.M., Giannobile, W.V. \& Kirkwood, K.L. (2007) Actinobacillus actinomycetemcomitans lipopolysaccharide-mediated experimental bone loss model for aggressive periodontitis. Journal of Periodontology 78: 550558.

Schwarz, F., Iglhaut, G. \& Becker, J. (2012) Quality assessment of reporting of animal studies on pathogenesis and treatment of peri-implant mucositis and peri-implantitis. A systematic review using the ARRIVE guidelines. Journal of Clinical Periodontology 39(Suppl 12): 63-72.

Trombelli, L., Farina, R., Marzola, A., Bozzi, L., Liljenberg, B. \& Lindhe, J. (2008) Modeling and remodeling of human extraction sockets. Journal of Clinical Periodontology 35: 630-639.

Vignoletti, F. \& Abrahamsson, I. (2012) Quality of reporting of experimental research in implant dentistry. Critical aspects in design, outcome assessment and model validation. Journal of Clinical Periodontology 39(Suppl 12): 6-27. 